This is a PDF file of an unedited manuscript that has been accepted for publication. Final edited form is published in J Magn Magn Mater, 2015, 380:144-149. http://dx.doi.org/10.1016/j.jmmm.2014.10.091

\title{
Controlled clustering of carboxylated SPIONs through polyethylenimine
}

\author{
Dániel Nesztor, Krisztina Bali, Ildikó Y. Tóth, Márta Szekeres, Etelka Tombácz*
}

Department of Physical Chemistry and Materials Science, University of Szeged, Aradi vértanúk tere 1, 6720, Szeged, Hungary

*Corresponding author: Etelka Tombácz (tel.:+3662544212; e-mail address: tombacz@chem.u-szeged.hu )

\section{Highlights}

- SPION clusters of controlled size were prepared by means of electrostatic adhesion.

- Nanocluster formation optimum was at 0.17 charge ratio of PEI to PAM@MNP.

- Huge aggregates form at higher PEI to PAM@MNP charge ratio.

- Higher ionic strength promotes the formation of clusters at lower PEI concentrations.

\begin{abstract}
Clusters of magnetite nanoparticles (MNPs) were synthesized using poly(acrylic acid-co-maleic acid) coated MNPs (PAM@MNP) and branched polyethylenimine (PEI). Materials were characterized by potentiometric titration, zeta potential and dynamic light scattering (DLS) measurements. PEI and PAM@MNP are oppositely charged as characterized by zeta potential measurements $(+8,-34 \mathrm{mV}$

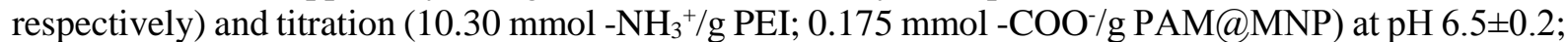
therefore magnetic clusters are formed by electrostatic adhesion. Two different preparation methods and the effect of PEI and electrolyte $(\mathrm{NaCl})$ concentration on the cluster formation was studied. Choosing an optimal concentration of PEI (charge ratio of PEI to PAM@MNP: 0.17) and electrolyte (10 mM), a concentrated (10 g MNP/L) product containing PEI-PAM@MNP nanoclusters with size of $165 \pm 10 \mathrm{~nm}$ was prepared. Its specific absorption rate (SAR) measured in AC magnetic field $(110 \mathrm{kHz}, 25 \mathrm{mT})$ is 12 $\mathrm{W} / \mathrm{g} \mathrm{Fe}$. The clustered product is expected to have enhanced contrast efficiency in MRI.
\end{abstract}

Keywords: superparamagnetic iron oxide nanoparticle, magnetic fluid, carboxylated SPION, magnetic cluster, hyperthermia, specific absorption rate.

\section{Introduction}

The application of nanoparticles in medicine has been in the focus over the past few decades. Including magnetic nanoparticles which are promising materials for use in magnetic resonance imaging (MRI) diagnostics, magnetic fluid hyperthermia, drug delivery or controlled drug release [1-5]. The most advanced approach is to combine diagnostics and therapy (theranostics) making clinical applications more efficient. The surface of magnetite nanoparticles (MNPs) has to be coated to prevent aggregation at physiological conditions (neutral pH, high electrolyte concentration) [6-9]. Metal-oxide surfaces are believed to form complexes with molecules having carboxylic moieties such as carboxylated polyelectrolytes (PE) that are a good choice for covering the surface [10-13]. The adsorbed polyelectrolyte layer stabilizes the particles both in an electrostatic and steric manner (electrosteric) [14].

The growing interest in the use of superparamagnetic iron oxide nanoparticles (SPIONs) for biomedical purposes indicates the need for products with enhanced properties which may possess greater potential in theranostics. Recent studies indicate that there is little to no cytotoxicity of SPIONs which makes them a promising candidate for biomedical applications [4, 7, 8, 15-18].

Magnetic fluid hyperthermia is also one of the key areas where such products may be utilized. During a hyperthermia treatment magnetic fluid is dispersed in the target tissue and the particular area is subjected to an alternating magnetic field to damage malignant cells through heat generation. The power absorbed and in turn dissipated by the different magnetic fluids is compared using the SAR value (specific absorption rate), that is the power absorbed per gram of magnetic material in the sample $(\mathrm{W} / \mathrm{g})$.

$$
S A R=\frac{C_{p, s} \rho}{\phi} \frac{\Delta T}{\Delta t}\left(=\frac{\sum_{i} C_{p i} m_{i}}{m_{F e}} \frac{\Delta T}{\Delta t}\right)
$$


where $C_{p, s}=\sum_{i} C_{p i} m_{i}$ is the specific heat capacity of the sample $(\mathrm{J} / \mathrm{Kg}), \rho$ is the density of the sample $\left(\mathrm{g} / \mathrm{cm}^{3}\right), \phi$ is the concentration of iron in the sample $\left(\mathrm{g} / \mathrm{cm}^{3}\right)$ and $\Delta T / \Delta t$ is the rate of temperature change $(\mathrm{K} / \mathrm{s})$ measured experimentally. Various forms of the formula for calculating SAR are presented in the literature [19-22], some sources calculate SAR per gram of iron-oxide (e.g. magnetite) or approximate the specific heat capacity of the sample with that of water.

Recently Illés et al. published that clustered PEG-OA coated magnetite nanoparticles showed superior MRI contrast to the non-clustered product [9]. The magnetic behavior of the particles in such clusters can be altered by particle-particle interactions [23-25]. It is crucial to understand the relation between nano-sized domains, and control particle-particle interactions to develop methods of particle fabrication and self-assembly. Direct manipulation of the interparticle spacing, thus the interaction, could help structure new materials with tailored properties. The non-covalent interaction between particles can be used to induce self-assembly formation and create nanostructures via the "bottom-up" fabrication [26]. Several methods have been presented to synthesize nanoclusters [27-28], but the electrostatics, i.e. the charge state of the individual building blocks is often neglected. Frankamp et al used polyamidoamine (PAMAM) dendrimers to assemble particles using electrostatic interactions and based on the dendrimer generation an increase of interparticle spacing was observed [29]. We aimed to control the self-assembly of particles not only by considering electrostatics, but by precisely determining the charge state of the interacting entities, thus controlling the prompt and irreversible interaction between oppositely charged particles. Our goal was to prepare magnetic clusters by electrostatic adhesion of negatively charged carboxylated MNPs and positively charged branched polyelectrolyte. We aimed to adjust the properties of the products to be colloidally stable at physiological $\mathrm{pH}$ and salt concentrations and also resist dilution. We wished to test the products in MF hyperthermia and later in MRI diagnostics, too.

\section{Materials and methods}

\subsection{Materials}

Magnetite nanoparticles (MNPs) were synthesized by the co-precipitation of iron salts of $\mathrm{FeCl}_{3} \cdot 6 \mathrm{H}_{2} \mathrm{O}$ and $\mathrm{FeCl}_{2} \cdot 4 \mathrm{H}_{2} \mathrm{O}$ (Molar, Hungary) with concentrated $\mathrm{NaOH}$ solution. Detailed description can be found in our previous paper [7]. The purified stable magnetic sol was stored at $\mathrm{pH} 3 \pm 0.2$ and temperature $4 \pm 1^{\circ} \mathrm{C}$. Transmission electron microscopy images revealed that the nanoparticles are spherical with the average size of $10 \pm 0.8 \mathrm{~nm}$ and the system shows low degree of polydispersity.

Poly(acrylic acid-co-maleic acid) (PAM) ( $\left.\mathrm{M}_{\mathrm{w}} \sim 1.8 \mathrm{kDa}, \mathrm{mmol} \mathrm{COOH} / \mathrm{g}\right)$ and branched polyethylenimine $(\mathrm{PEI})\left(\mathrm{M}_{\mathrm{w}} \sim 25 \mathrm{kDa}\right)$ were acquired from Sigma-Aldrich. $\mathrm{NaCl}, \mathrm{NaOH}$ and $\mathrm{HCl}$ solutions prepared from analytical grade chemicals (Molar, Hungary) were used to set ionic strength and the $\mathrm{pH}$, respectively. Ultra pure (UP) water (18 M $\Omega$ ) was provided by a Zeneer (HumanCorp, Korea) water purification system. All experiments were conducted at $25 \pm 1{ }^{\circ} \mathrm{C}$.

\subsection{Preparation of PAM coated magnetite nanoparticles}

The core shell nanoparticles were prepared by adding PAM $\left(1.1 \mathrm{mmol}-\mathrm{COO}^{-} / \mathrm{g} \mathrm{MNP}\right)$ to a portion of the magnetic fluid we synthesized earlier. The amount of PAM is given regarding the amount of carboxylic groups $(\mathrm{mmol} \mathrm{COO} / \mathrm{COOH})$ because the molecular weight of PAM is an average value of the polydisperse sample. The sample contained $0.6 \mathrm{~g}$ of MNPs and the adsorption of PAM commenced at $\mathrm{pH} 6.5 \pm 0.2$ and at an ionic strength of $10 \mathrm{mM}(\mathrm{NaCl})$. The mixture was rested for 24 hours for the adsorption to complete The excess of PAM (adsorption maximum: $0.9 \mathrm{mmol} / \mathrm{g}$ MNP [7]) was removed by a washing process that was repeated 3 times: the stabilizing PAM layer was compacted by lowering the $\mathrm{pH}$ to 3 and the aggregated SPIONs were settled by using a strong magnet that was placed under the vial; the supernatant was removed and the nanoparticles were re-dispersed in UP water. The $\mathrm{pH}$ was set to 6.5 and the resulting stable PAM@MNP sol (42.85 g MNP/L) was stored in a refrigerator $\left(4 \pm 1^{\circ} \mathrm{C}\right)$.

\subsection{Preparation of PEI-PAM@MNP nanoclusters}

The nanoclusters were prepared by adding PEI to the PAM@MNP sol in different amounts, hypothesizing an electrostatic structural self-assembly due to attraction between oppositely charged partners (Fig. 1). The effect of PEI to PAM@MNP ratio and $\mathrm{NaCl}$ concentration on the cluster formation 
was investigated. By increasing the concentration of PEI the negative charge of the PAM@MNP particles is gradually compensated then overcompensated. The clusters were prepared pursuing two different methods: the fast flow and the synchronous flow method. In both of the methods identical portions $(5-5 \mathrm{~mL})$ of PEI solution and PAM@MNP $(300 \mathrm{mg} / \mathrm{L})$ sol were mixed. In the fast flow method a tilted $\left(45^{\circ}\right)$ vial containing $5 \mathrm{~mL}$ of PAM@MNP sol was held in ultrasonic bath and after 2 minutes of sonication $5 \mathrm{~mL}$ of PEI was promptly injected to the bottom edge of the vial using a syringe and the mix was sonicated to an additional time of 1 minute. During the preparation of clusters through the synchronous flow method the two precursors were simultaneously and promptly injected into a vial and sonicated for 1 minute immediately after mixing (Fig. 2).

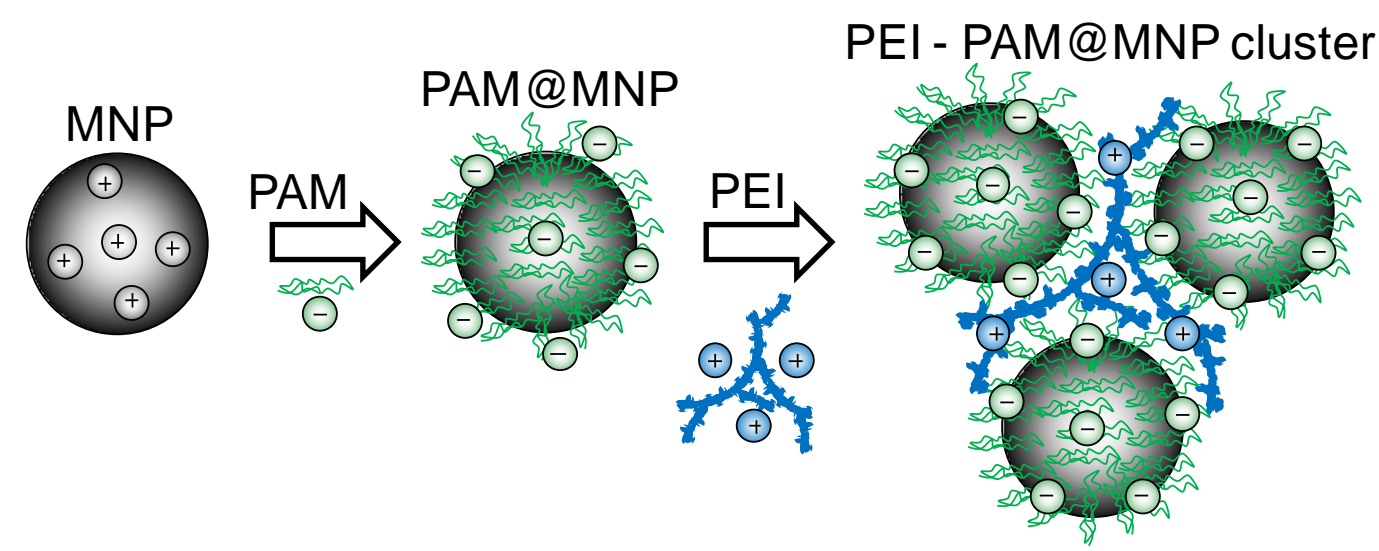

Fig. 1. Electrostatic adhesion of negatively charged poly(acrylic acid-co-maleic acid) coated magnetite nanoparticles and positively charged polyethylenimine at $\mathrm{pH} 6.5 \pm 0.2$

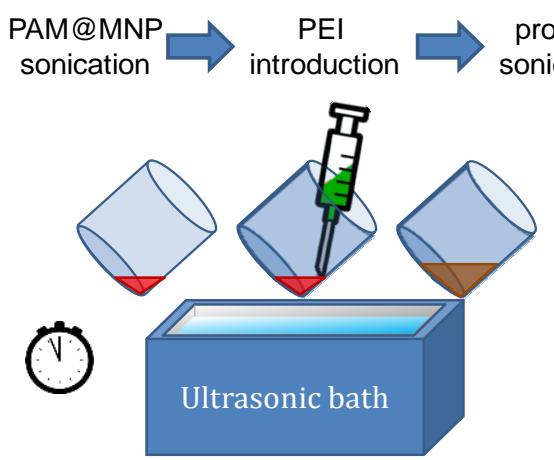

Fast flow method

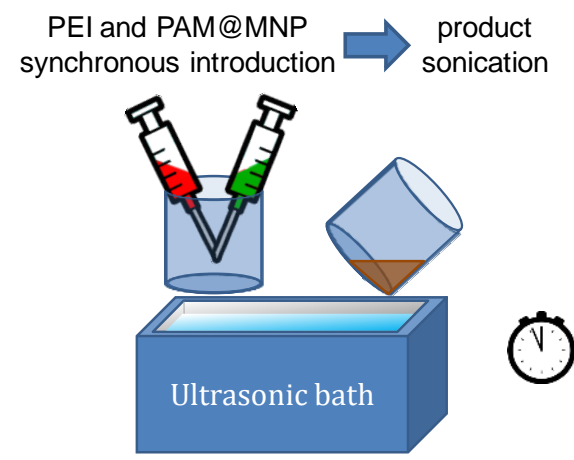

Synchronous flow method

Fig. 2. Preparation of clusters: the fast flow (left) and the synchronous flow method (right)

\subsection{Potentiometric acid-base titration measurements}

Potentiometric acid-base titration of PEI and PAM@MNP samples $(50 \mathrm{~mL}$ of $2.60 \mathrm{~g} \mathrm{PEI} / \mathrm{L}$ and $2.80 \mathrm{~g} \mathrm{MNP/L}$ respectively) was performed to determine their charge state. The procedure was described previously [30]. The background electrolyte, $\mathrm{NaCl}$ (was indifferent with no specific interactions of its ions with either of the materials. Samples were titrated at 3 different ionic strengths: 5,50 and, $500 \mathrm{mM}$. The data points of the titration results express equilibrium states. The equilibrium criterion of $\Delta \mathrm{pH} / \mathrm{min}$ $<0.01$ was used.

\subsection{Dynamic light scattering and electrophoretic mobility measurements}

Dynamic light scattering (DLS) and electrophoretic mobility measurements of the samples were carried out in a NanoZS instrument (Malvern, UK) with a $4 \mathrm{~mW} \mathrm{He}-\mathrm{Ne}$ laser source $(\lambda=633 \mathrm{~nm})$, using disposable zeta cells (DTS 1060). The concentration of the dispersions was set to give an optimal 
intensity of $\sim 10^{5}$ counts per second. Prior to the measurements, the samples were agitated with ultrasound for $10 \mathrm{~s}$ and allowed to relax for $2 \mathrm{~min}$. The effect of PEI to PAM@MNP charge ratio on the electrophoretic mobility and particle size of the MNPs was measured at $\mathrm{pH} 6.5 \pm 0.2$ and $10 \mathrm{mM}$ ionic strength. The Smoluchowski equation was applied to convert the electrophoretic mobilities to electrokinetic potential values.

\subsection{Magnetic fluid hyperthermia measurements}

The heating efficiency of concentrated magnetic fluid samples was tested in the magneTherm ${ }^{\mathrm{TM}}$ (Nanotherics, UK) system for hyperthermia. Preceding the measurement of the nanocluster sample the effect of the applied alternating magnetic field was tested on naked MNP samples, by employing different pairs of coils and capacitors resulting in unique resonant frequencies optimal settings were determined. The effect of the concentration of magnetic sols containing naked MNPs was also studied. Heating efficiency of the concentrated nanocluster sample was measured at a resonant frequency of $110.7 \mathrm{kHz}$ with magnetic field of $\mathrm{B}=25 \mathrm{mT}(\mathrm{H}=19.9 \mathrm{kA} / \mathrm{m}, 17$ turn coil and $200 \mathrm{nF}$ capacitor $)$ and concentration range of 10-15 g MNP/L. SAR values were calculated according to Eq. 1 with the sample specific heat capacity.

\section{Results}

\subsection{Characterization of the interacting partners}

Polyethylenimine is a branched and dendrimer like polyelectrolyte having amine groups $\left(-\mathrm{NH}_{2} /-\right.$ $\left.\mathrm{NH}_{3}{ }^{+}\right)$that can be protonated at neutral $\mathrm{pH}$. The monomer of PEI $\left(\mathrm{M}_{\mathrm{w}} \sim 474 \mathrm{~g} / \mathrm{mol}\right)$ has 4 primary, 3 secondary and 4 tertiary amine groups. On the other hand PAM@MNP has carboxyl groups (-COOH) that undergo deprotonation $\left(-\mathrm{COO}^{-}\right)$at higher $\mathrm{pH}$ values. This results in the macromolecule to be negatively charged. The adsorbed polyanion binds to the surface of magnetite nanoparticles with high affinity and provides both steric and electrostatic stabilization. In our previous work, the mechanism of MNP coating via adsorption of PAM has been studied in detail [7]. The pH-dependent protonationdeprotonation processes can be characterized quantitatively by potentiometric acid-base titration [30]. The net proton surface excess amounts calculated from the titration data of PEI solution and PAM@MNP sol are shown as a function of $\mathrm{pH}$ in (Fig. 3). It is obvious that the measured samples are oppositely charged above $\mathrm{pH} \sim 6$. At $\mathrm{pH} 6.5$, the primary amino groups of PEI are protonated (10.30 mmol $-\mathrm{NH}_{3}{ }^{+}$per gram of PEI at $\left.50 \mathrm{mM} \mathrm{NaCl}\right)$ and the carboxyl groups of PAM@MNP are deprotonated (0.175 mmol -COO- per gram of PAM@MNP at $50 \mathrm{mM})$. The net proton surface excess of PAM@MNP is positive at lower $\mathrm{pH}$ values which can be explained by the diffusion of protons through the PAM shell to the surface, resulting in the protonation of the free surface sites of magnetite $\left(\equiv \mathrm{Fe}-\mathrm{OH}+\mathrm{H}^{+} \rightarrow \equiv \mathrm{Fe}-\right.$ $\mathrm{OH}_{2}{ }^{+}$). The neutralization of PAM@MNP surface charge by PEI was calculated (PEI to PAM@MNP charge ratio) at each PEI addition during the nanocluster preparation.

The charge state of the two interacting partners was also investigated by electrophoretic mobility measurements and the resulting data of zeta potential at different $\mathrm{pHs}$ also proved that electrostatic adhesion of PEI and PAM@MNP is possible. The zeta potential of PEI changed from 15 to $5 \mathrm{mV}$ in between $\mathrm{pH}$ of 3 and 10, while the hydrodynamic size remained the same (14-15 nm) (Fig. 4). The zeta potential of PAM@MNP decreased from 0 to $-35 \mathrm{mV}$ in between $\mathrm{pH}$ of 3 and 10 . The hydrodynamic size also decreased from $\sim 1500$ to $\sim 120 \mathrm{~nm}$ in this range, showing that aggregation of the particles occurs at $\mathrm{pH}$ values lower than 4 (Fig. 4). This means that the positively charged PEI is able to interact with the negatively charged PAM@MNP particles and form clusters spontaneously through multipoint electrostatic adhesion. The amount of PEI needed to induce the formation of clusters was calculated by using the concentration of charged carboxylate (-COO-) groups on PAM@MNP at pH 6.5 \pm 0.2 . 

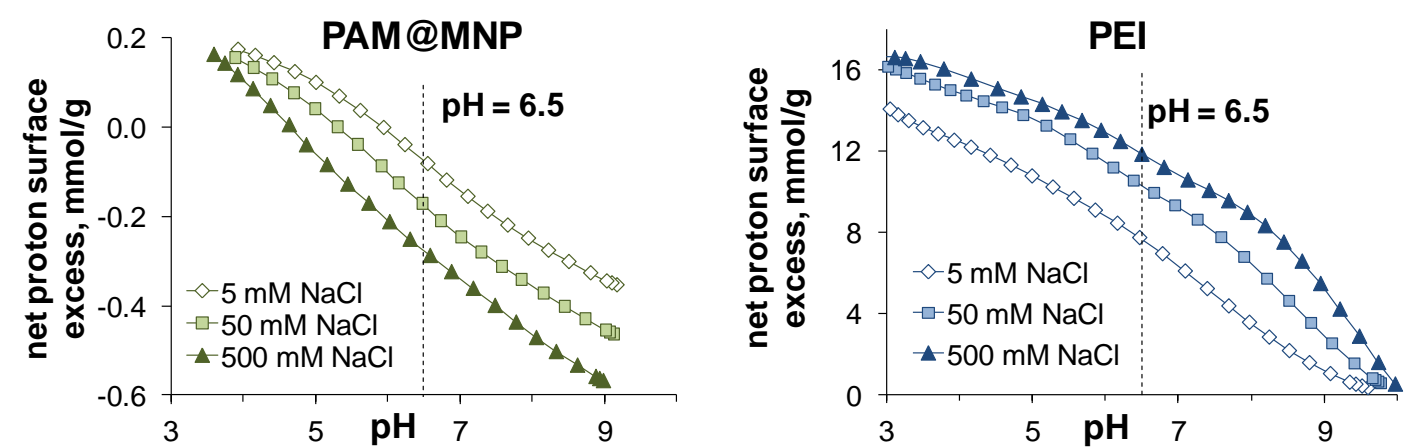

Fig. 3. $\mathrm{pH}$ - and ionic strength-dependent charging of poly(acrylic acid-co-maleic acid) coated magnetite nanoparticles (left) and polyethylenimine (right). The net proton surface excess amount is proportional to and can be converted directly to surface charge density data.
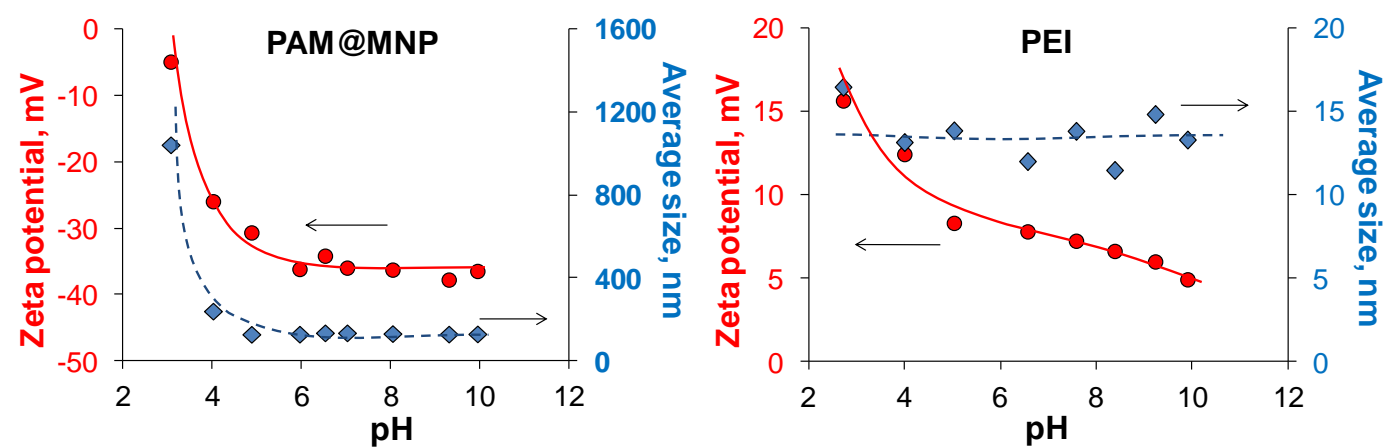

Fig. 4. pH-dependence of average hydrodynamic size (diamond symbols) and zeta potential (circle symbols) of poly(acrylic acid-co-maleic acid) coated magnetite nanoparticles (left) and polyethylenimine (right) at ionic strength of $10 \mathrm{mM}$

\subsection{Effect of PEI to PAM@MNP ratio on the formation of clusters}

The concentration of PEI added to the constant amount of PAM@MNP sols and $\mathrm{NaCl}$ content of the mixture were changed. The charge ratio of PEI to PAM@MNP was varied up to 1.5 and the obtained products were characterized by DLS and zeta potential measurements. We have found that at very low PEI concentrations there is no significant change in hydrodynamic size $(125 \mathrm{~nm})$ even though the zeta potential increases slightly compared to PAM@MNP particles ( -35 to $-30 \mathrm{mV}$ ) (Fig. 5). The increase in zeta potential could be attributed to the charge neutralization that occurs on individual particles of PAM@MNP just before there is enough PEI to adhere these individual particles forming clusters. At slightly higher PEI concentrations there is an increase in the size (125 to $300 \mathrm{~nm})$ and decrease in the zeta-potential of particles ( -30 to $-35 \mathrm{mV}$ ), which indicate the formation of clusters that now exhibit an overall negative charge. From this point on with increasing PEI concentrations the zeta potential increases ( -35 to $-25 \mathrm{mV}$ ) and the increase in the size of the particles (from 300 to $1800 \mathrm{~nm}$ ) shows that aggregates are formed (Fig. 5). At about 0.6 PEI to PAM@MNP charge ratio the aggregate size reaches a plateau. With even higher concentrations of polyethylenimine the zeta potential changes from negative to positive (-25 to $+5 \mathrm{mV}$ ) which is consistent with the overcompensating charge ratio (1.5) caused by PEI (Fig. 5). 


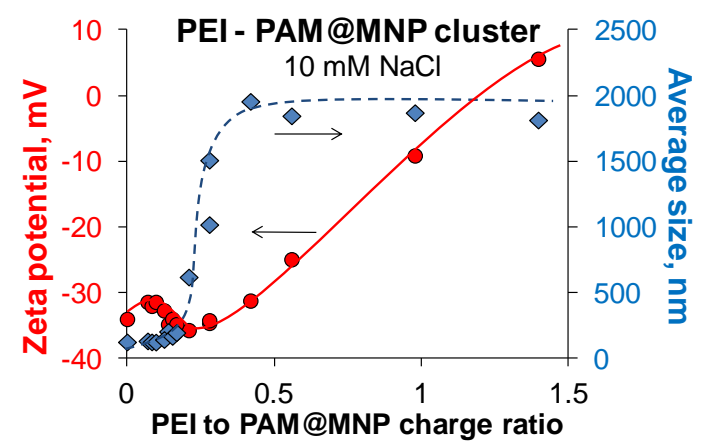

Fig. 5. Average hydrodynamic size (diamond symbols) and particle charge (circle symbols) of PEI-PAM@ MNP clusters formed with increasing charge compensation (concentration) of $5 \mathrm{~mL}$ PEI solution added to $5 \mathrm{~mL}$ of 300 mg/L PAM@MNP sols. Dynamic light scattering (DLS) and zeta potential of prepared clusters were measured at ionic strength of $10 \mathrm{mM}$ and $\mathrm{pH} \sim 6.5$

\subsection{Influence of preparation methods on cluster formation}

We hypothesized that the methods of nanocluster preparation (fast flow and synchronous flow) and the rate of flow from the syringes would have an impact on the formation of nanoclusters due to the prompt and irreversible interaction between particles of opposite charge. Thus the effect of volumetric flow rate of the PEI solution was investigated using the fast flow method in such a manner that the syringes used for the preparation were equipped with two different needle sizes: $18 \mathrm{G}$ and $25 \mathrm{G}$ with $0.838 \mathrm{~mm}$ and $0.260 \mathrm{~mm}$ inner diameters, respectively. The nanoclusters prepared by employing the two different needles did not differ substantially in size and zeta potential (presented in supplementary material, Fig. S2.). This showed that there is no effect with increasing the flow rate, but for the convenience of prompt injection from the syringes the $18 \mathrm{G}$ needles were used for further tests. Comparison of the two methods used to prepare nanoclusters indicated that the the fast flow method caused the formation of slightly larger clusters at a PEI to PAM@MNP charge ratio compared to the synchronous flow method but practically resulting in the same zeta potential (Fig. 6). Although this difference did not seem significant, the more moderate and controllable growth of the particle size with increasing PEI concentrations led us to choose the synchronous flow method of preparation for further tests. A possible explanation to why neither the methods nor the needle size had substantial effect would be that the applied sonication provided a uniform continuous dispersing throughout the mixture leading to very similar kinetics of nanocluster formation.

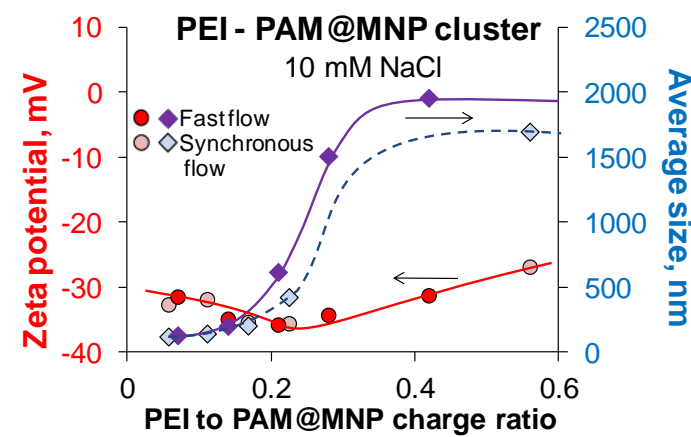

Fig. 6. Influence of preparation methods (Fast flow - filled symbols and synchronous flow - half-filled symbols) on cluster formation: dynamic light scattering and zeta potential measurements at $\mathrm{pH} 6.5 \pm 0.2$

\subsection{Effect of ionic strength on the formation of clusters}

The ionic strength ( $\mathrm{NaCl}$ salt concentration) was $10 \mathrm{mM}$ throughout the synthesis of PAM@MNP and also when investigating the effect of PEI concentrations, needle size and method of preparation used. We expected the ionic strength to be a key aspect when preparing the clusters, so we examined the effect choosing $30 \mathrm{mM}$ and $100 \mathrm{mM}$ of $\mathrm{NaCl}$ salt concentrations with the already determined optimal PEI concentrations. The effect we observed was significant, the formation of the clusters and aggregates began at markedly lower concentrations of PEI also, leading to a steeper rise and faster plateauing of the particle size (150, 500 and $1500 \mathrm{~nm}$ at 0.15 PEI to PAM@MNP charge ratio 
at a concentration of $\mathrm{NaCl}$ of 10,30 and $100 \mathrm{mM}$ respectively) (Fig. 7). This result is consistent with the charge screening effect of the ionic strength around particles leading to an easier formation of aggregates. The tenfold dilution of samples prepared at $100 \mathrm{mM}$ ionic strength showed a much more moderate increase in particle size compared to the samples prepared at $10 \mathrm{mM}$ meaning that the dilution of the formed aggregates resulted in nanoclusters of smaller size (Fig. 8).

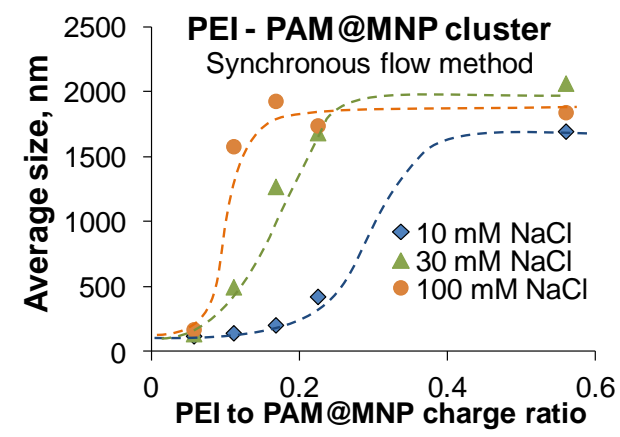

Fig. 7. Effect of ionic strength: dynamic light scattering measurements of prepared clusters at $\mathrm{pH} 6.5 \pm 0.2$ using the synchronous flow method

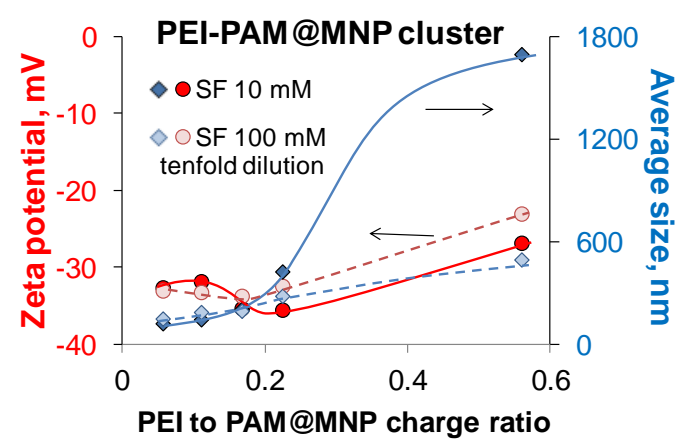

Fig. 8. Comparison of nanoclusters prepared with the synchronous flow method at $10 \mathrm{mM}$ and at the tenfold dilution of $100 \mathrm{mM}$ : dynamic light scattering and zeta potential measurements at $\mathrm{pH} 6.5 \pm 0.2$

\subsection{Preparation of concentrated PEI-PAM@MNP sol}

The investigation of several effects resulted in optimal parameters that were chosen to prepare a concentrated PEI-PAM@MNP nanocluster sol. We chose a salt concentration of $10 \mathrm{mM}$ and a PEI concentration corresponding to 0.17 PEI to PAM@MNP charge ratio. The concentrated magnetic fluid containing nanoclusters was prepared employing the synchronous flow method with the $18 \mathrm{G}$ needle. The size and zeta potential of the prepared product was measured after diluting the concentrated sol and setting the ionic strength to $10 \mathrm{mM}$. The results indicate that nanoclusters with a hydrodynamic diameter of $165 \pm 10 \mathrm{~nm}$ were formed that show good stability over the period of days (presented in supplementary material, Fig. S1).

\subsection{Hyperthermia results}

The heating effect of PEI-PAM@MNP nanoclusters was tested in the concentrated sol as prepared and was compared to the result obtained for the MFs containing naked MNPs, the calculated SAR values related to unit mass of iron were very similar. Although there is a slight increase in SAR in favor of the nanoclusters it is within statistical error of the measurement (Fig. 9). The SAR values are small but in the order of magnitude found in the literature, since the reported values are in a wide range of 1.6-2055 W/g [22].This indicates either a poor choice of the optimal parameters of the hyperthermia test concerning the nanoclusters or that there is no additional effect of heating when compared to the naked MNPs. Further tests are necessary to conclude whether the nanocluster product is superior to naked MNPs. 


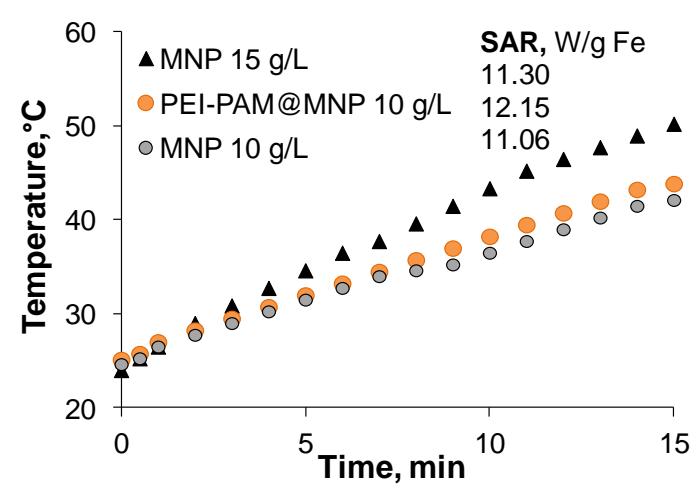

Fig. 9. Hyperthermia test of magnetic fluids containing naked magnetite nanoparticles and PEI-PAM@MNP clusters in a magnetic field of $B=25 \mathrm{mT}$ and resonant frequency of $\mathrm{f}=111 \mathrm{kHz}$

\section{Conclusions}

Nanoclusters were prepared using the electrostatic adhesion between negatively charged PAM coated magnetite particles and the positively charged branched PEI. It was shown that with increasing the PEI to PAM@MNP ratio the hydrodynamic size of the clusters can be controlled to the extent where obvious aggregation of the particles occurs. The ionic strength has a great impact on the formation of clusters by providing an electrostatic screening effect that promotes the formation of nanoclusters and aggregates at significantly lower PEI concentrations. The two methods of preparation (fast flow and synchronous flow) as well as changing the rate of flow through employing needles of different diameter showed no significant effect on the measured size and zeta potential of the particles, probably because of continuous sonication during the mixing the oppositely charged interacting partners.

A concentrated sol of magnetic clusters was prepared under the optimal conditions of nanocluster formation for testing its hyperthermia efficiency. The results show that there is only a slight increase in the SAR value in favor of the nanoclusters indicating that further tests to optimize the conditions of field exposure are necessary or clusters made up of larger size individual MNPs need to be prepared. The product has not been tested in MRI diagnostics, nevertheless enhanced contrast efficiency (through increased $\mathrm{r} 2$ relaxivity) of the clustered MNPs is expected [9].

\section{Acknowledgements}

This work was supported by the OTKA Foundation (NK84014).

\section{References}

[1] Q.A. Pankhurst, N.K.T. Than, S.K. Jones, J. Dobson, Progress in applications of magnetic nanoparticles in biomedicine, J. Phys. D Appl. Phys. 42 (2009) art. no. 224001

[2] O. Veiseh, J.W. Gunn, M. Zhang, Design and fabrication of magnetic nanoparticles for targeted drug delivery and imaging, Adv. Drug Deliv. Rev. 62 (2010) 284-304.

[3] J.K. Oh, J.M. Park, Iron oxide-based superparamagnetic polymeric nanomaterials: design, preparation, and biomedical application, Prog. Polym. Sci. 36 (2011) 168-189.

[4] E.J. Rosen, L. Chan, D.-B. Shieh, F.X. Gu, Iron oxide nanoparticles for targeted cancer imaging and diagnostics, Nanomed.-Nanotechnol. 8 (2012) 275-290.

[5] C. Corot, D. Warlin, Superparamagnetic iron oxide nanoparticles for MRI: contrast media pharmaceutical company R\&D perspective, Wiley Interdiscip. Rev. Nanomed. Nanobiotechnol. 5 (2013) 411-422.

[6] D. Bica, L. Vékás, M.V. Avdeev, O. Marinica, V. Socoliuc, M. Balasoiu, V.M. Garamus, Sterically stabilized water based magnetic fluids: synthesis, structure and properties, J. Magn. Magn. Mater. 311 (2007) 17-21. 
This is a PDF file of an unedited manuscript that has been accepted for publication. Final edited form is published in J Magn Magn Mater, 2015, 380:144-149. http://dx.doi.org/10.1016/j.jmmm.2014.10.091

[7] I.Y. Tóth, E. Illés, R.A. Bauer, D. Nesztor, M. Szekeres, I. Zupkó, E. Tombácz, Designed polyelectrolyte shell on magnetite nanocore for dilution-resistant biocompatible magnetic fluids, Langmuir 28 (2012) 16638-16646.

[8] M. Szekeres, I.Y. Tóth, E. Illés, A. Hajdú, I. Zupkó, K. Farkas, G. Oszlánczi, L.Tiszlavicz, E. Tombácz, Chemical and colloidal stability of carboxylated core-shell magnetite nanoparticles designed for biomedical applications, Int. J. Mol. Sci. 14 (2013) 14550-14574.

[9] E. Illés, M. Szekeres, E. Kupcsik, I.Y. Tóth, K. Farkas, A. Jedlovszky-Hajdú, E. Tombácz, PEGylation of surfacted magnetite core-shell nanoparticles for biomedical application, Colloid. Surface A 460 (2014) 429-440.

[10] M. Kumagai, Y. Imai, T. Nakamura, Y. Yamasaki, M. Sekino, S. Ueno, K. Hanaoka, K. Kikuchi, T. Nagano, E. Kaneko, K. Shimokado, K. Kataoka, Iron hydroxide nanoparticles coated with poly(ethylene glycol)-poly(aspartic acid) block copolymer as novel magnetic resonance contrast agents for in vivo cancer imaging, Colloid. Surface B 56 (2007) 174-181.

[11] K. Vermöhlen, H. Lewandowski, H.-D. Narres, E. Koglin, Adsorption of polyacrylic acid on aluminium oxide: DRIFT spectroscopy and ab initio calculations, Colloid. Surface A 170 (2000) 181-189.

[12] H. Hu, J. Saniger, J. Garcia-Alejandre, V.M. Castaño, Fourier transform infrared spectroscopy studies of the reaction between polyacrylic acid and metal oxides, Mater. Lett. 12 (1991) 281-285.

[13] J. Lyklema, L. Deschênes, The first step in layer-by-layer deposition: Electrostatics and/or nonelectrostatics?, Adv. Colloid Interfac. 168 (2011) 135-148.

[14] M.A. Cohen Stuart, Polyelectrolyte adsorption, J. Phys. France 49 (1988) 1001-1008.

[15] M. Mahdavi, M.B. Ahmad, M.J. Haron, F. Namvar, B. Nadi, M.Z.A. Rahman, J.Amin, Synthesis, surface modification and characterisation of biocompatiblemagnetic iron oxide nanoparticles for biomedical applications, Molecules 18 (2013) 7533-7548.

[16] L. Sun, C. Huang, T. Gong, S. Zhou, A biocompatible approach to surface modification: Biodegradable polymer functionalized super-paramagnetic iron oxide nanoparticles, Mater. Sci. Eng. C 30 (2010) 583-589.

[17] U.I. Tromsdorf, O.T. Bruns, S.C. Salmen, U. Beisiegel, H. Weller, A highly effective, nontoxic T1 MR contrast agent based on ultrasmall PEGylated iron oxide nanoparticles, Nano Lett. 9 (2009) 4434-4440.

[18] A. Masoudi, H.R.M. Hosseini, M.A. Shokrgozar, R. Ahmadi, M.A. Oghabian, The effect of poly(ethylene glycol) coating on colloidal stability of superparamagnetic iron oxide nanoparticles as potential MRI contrast agent, Int. J. Pharm. 433 (2012) 129-141.

[19] B. Mehdaoui, A. Meffre, J. Carrey, S. Lachaize, L.-M. Lacroix, M. Gougeon, B. Chaudret, M. Respaud, Optimal size of nanoparticles for magnetic hyperthermia: A combined theoretical and experimental study, Adv. Funct. Mater. 21 (2011) 4573-4581.

[20] S. Laurent, S. Dutz, U.O. Häfeli, M. Mahmoudi, Magnetic fluid hyperthermia: Focus on superparamagnetic iron oxide nanoparticles, Adv. Colloid Interfac. 166 (2011) 8-23.

[21] G. Vallejo-Fernandez, O. Whear, A.G. Roca, S. Hussain, J. Timmis, V. Patel, K. O'Grady, Mechanisms of hyperthermia in magnetic nanoparticles, J. Phys. D Appl. Phys. 46 (2013) art. no. 312001

[22] C. Grüttner, K. Müller, J. Teller, F. Westphal, Synthesis and functionalisation of magnetic nanoparticles for hyperthermia applications, Int. J. Hyperther. 29 (2013) 777-789.

[23] B.L. Frankamp, A.K. Boal, M.T. Tuominen, V.M. Rotello, Direct control of the magnetic interaction between iron oxide nanoparticles through dendrimer-mediated self-assembly, J. Am. Chem. Soc. 127 (2005), 9731-9735.

[24] G. Herzer, Grain structure and magnetism of nanocrystalline ferromagnets, IEEE T Magn. 25 (1989) 3327-3329.

[25] J.L. Dormann, D. Fiorani, E. Tronc, Magnetic relaxation in fine-particle systems, Adv. Chem. Phys. 98 (1997) 283-494.

[26] A.K. Boal, F. Ilhan, J.E. Derouchey, T. Thurn-Albrecht, T.P. Russell, V.M. Rotello, Self-assembly of nanoparticles into structured spherical and network aggregates, Nature 404 (2000) 746-748. 
[27] A. Kukreja, E.-K. Lim, B. Kang, Y. Choi, T. Lee, J.-S. Suh, Y.-M. Huh, S. Haam, One-pot synthesis of magnetic nanoclusters enabling atherosclerosis-targeted magnetic resonance imaging. Int. J. Nanomed., 9 (2014) 2489-2498.

[28] Y. Qiang, J. Antony, A. Sharma, J. Nutting, D. Sikes, D. Meyer, Iron/iron oxide core-shell nanoclusters for biomedical applications. J. Nanopar. Res., 8 (2006) 489-496.

[29] B.L. Frankamp, A.K. Boal, V.M. Rotello, Controlled interparticle spacing through self-assembly of Au nanoparticles and poly(amidoamine) dendrimers, J. Am. Chem. Soc. 124 (2002) 1514615147.

[30] M. Szekeres, E. Tombácz, Surface charge characterization of metal oxides by potentiometric acidbase titration, revisited theory and experiment, Colloid. Surface A 414 (2012) 302- 313. 


\section{Supplementary material}

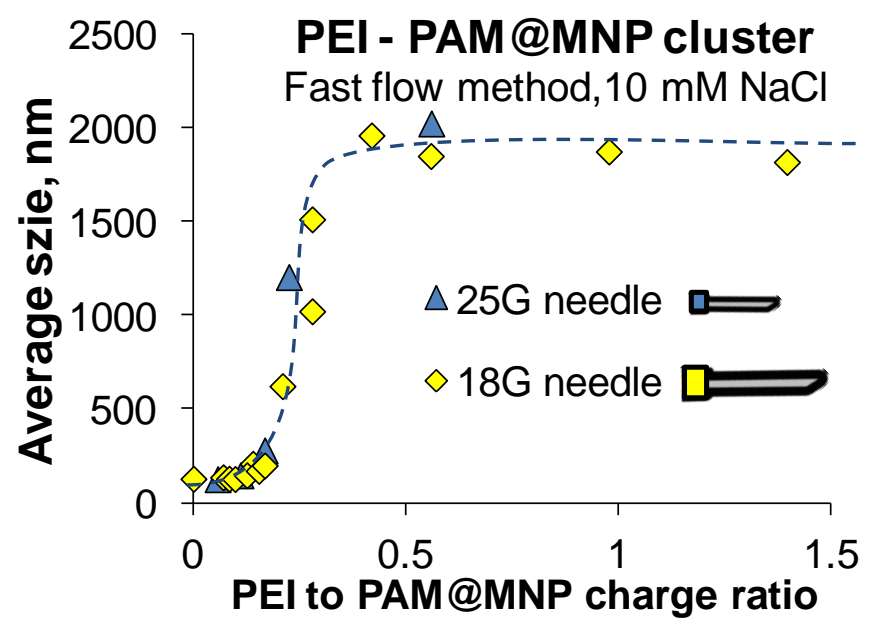

Figure S1. Effect of needle diameter on PEI-PAM@MNP cluster formation using the fast flow method: dynamic light scattering measurements of prepared clusters at $\mathrm{pH} 6.5 \pm 0.2$

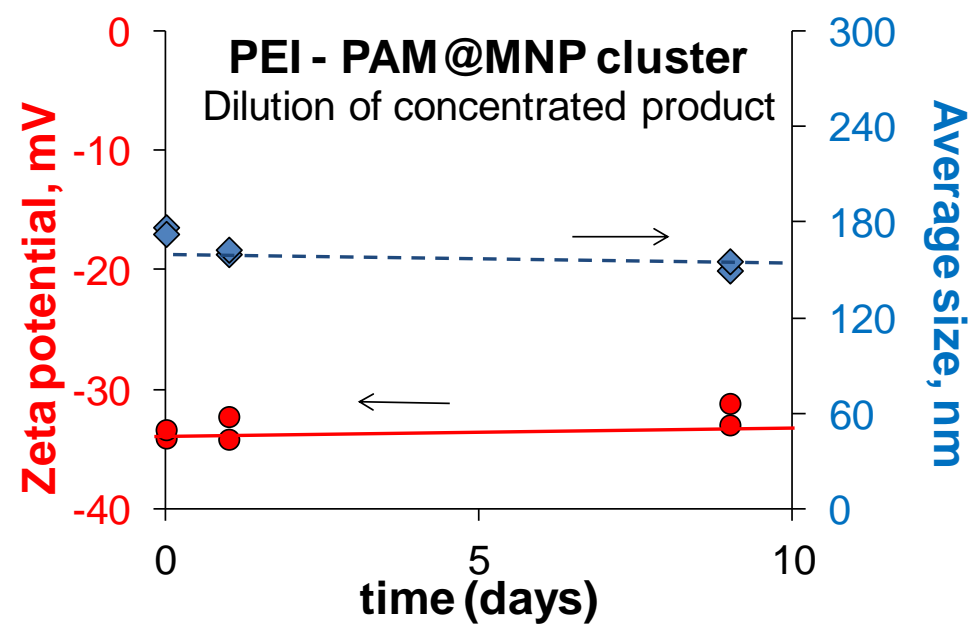

Figure S2. Time-stability of the PEI-PAM@MNP sample diluted from the concentrated product: dynamic light scattering and zeta potential measurements at $10 \mathrm{mM}$ and $\mathrm{pH} 6.5 \pm 0.2$ 